\title{
Influence de la nuance d'acier des roues ferroviaires en Fatigue de Contact de Roulement
}

\author{
Amavi Langueh ${ }^{1,3}$, Jean-François Brunel ${ }^{1,3}$, Eric Charkaluk ${ }^{1,2,4}$, Philippe Dufrenoy ${ }^{1,3}$ \\ et François Demilly ${ }^{5}$ \\ 1 Univ. Lille Nord de France, 59000 Lille, France \\ 2 ECLille, LML, 59650 Villeneuve d'Ascq, France \\ 3 USTL, LML, 59650 Villeneuve d'Ascq, France \\ ${ }^{4}$ CNRS, UMR 8107, 59650 Villeneuve d'Ascq, France \\ 5 GHH Valdunes, 59125 Trith Saint Leger, France
}

\begin{abstract}
Résumé. Cet article propose une méthodologie de prédiction de la durée de vie des roues ferroviaires permettant de prendre en compte les sollicitations locales via la géométrie réelle du contact roue/rail, le comportement inélastique du matériau (acier) et d'intégrer un critère de fatigue. Le contexte industriel, d'étudier l'influence de la nuance d'acier sur la durabilité de la roue. Les principales étapes de l'approche sont l'identification du comportement des matériaux, la détermination des champs de contraintedéformation stabilisés et l'application d'un critère de fatigue. L'algorithme stationnaire est utilisé pour déterminer les contraintes et déformations suivant les conditions d'exploitation. Trois aciers ont été étudiés en analysant leurs réponses mécaniques, leurs limites d'adaptation et leurs durées de vie moyenne.
\end{abstract}

\section{INTRODUCTION}

Avec l'augmentation des charges de transport à l'essieu, la maintenance des rails et des roues ferroviaires est devenu un enjeu économique majeur, au-delà de celui de la sécurité. La Fatigue de Contact de Roulement (FCR) est un phénomène d'endommagement qui apparaît sur les rails et les roues ferroviaires et qui est accélérée par l'augmentation des vitesses et des charges d'exploitation. On rencontre en général deux types de FCR dans les roues ferroviaires : l'endommagement de surface (shelling et spalling) et en sous-surface. Le diagramme d'adaptation et d'accommodation souvent appelé Shakedown map de Johnson [1] peut être utilisé pour déterminer le comportement asymptotique du matériau et donc le niveau de fatigue en fonction du chargement et des conditions de frottement. Les limites de la shakedown varient fortement avec le comportement mécanique du matériau (modèle élastique, parfaitement plastique, à écrouissage cinématique linéaire et non-linéaire).

La méthodologie de dimensionnement adoptée est détaillée dans les travaux de Langueh et al. [2].

\section{COMPORTEMENT DES MATÉRIAUX}

Les matériaux étudies sont le ER7 (XC60), le ER9 (XC50) et le 50CrMo4. Des essais d'écrouissage cyclique à déformation imposée ont été réalisés sur des éprouvettes prélevées à $15 \mathrm{~mm}$ sous la bande de roulement de la roue. Le modèle à écrouissage cinématique nonlinéaire d'Armstrong-Frédérick [3] a été utilisé pour décrire le comportement des matériaux. Les figures 1a, $1 \mathrm{~b}$ et 1c montrent la comparaison entre les cycles stabilisés contrainte-déformation numérique (obtenus avec l'algorithme génétique) et expérimentale respectivement pour les aciers ER7, ER9 et 50CrMo4. On constate un bon compromis entre entre ces deux types de courbes.

\section{SIMULATION NUMÉRIQUE DU CONTACT ROUE/RAIL}

L'algorithme stationnaire fondé sur la formulation eulérienne est utilisé. Cette méthode basée sur la configuration eulérienne permet de déterminer l'état stabilisé. Le modèle du contact roue/rail est présenté sur la figure 2 [2].

\section{Influence de la nuance d'aciers}

L'évolution des contraintes de von Mises en profondeur en conditions de roulement pur sous la bande de roulement pour les trois aciers est présentée sur la figure 3.

Pour chaque acier, l'évolution de de la contrainte de von Mises diffère et est liée à la déformation plastique. Le ER7 plastifie un peu à $20 \mathrm{kN}$ et le ER9 commence à plastifier à $50 \mathrm{kN}$. Quant au $50 \mathrm{CrMo} 4$, il n'y a pas de plastification à $20 \mathrm{kN}$, mais un début de l'écoulement plastique à 50 et $100 \mathrm{kN}$.

\section{Influence de la nuance d'aciers sur la réponse de la} structure

La réponse cyclique de la structure a été analysée le long de la ligne de courant contenant le point le plus sollicité en contrainte de cisaillement. La figure 4 représente les boucles contrainte/déformation obtenues pour des charges de 20,50 et $100 \mathrm{kN}$. 


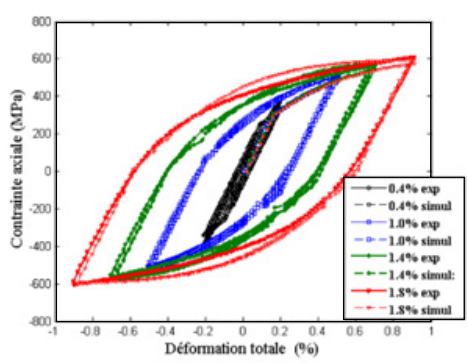

a.

b.
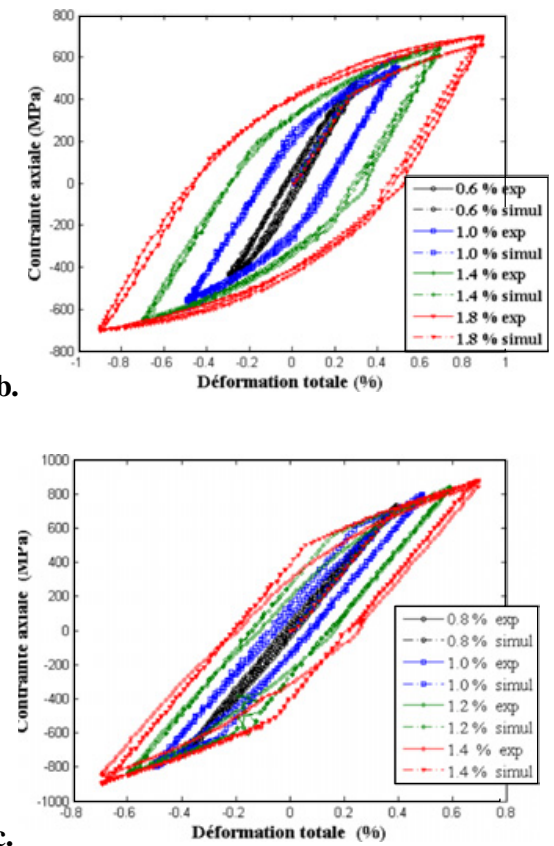

Figure 1. Boucle d'hystérésis stabilisées expérimentales : a (ER7), b (ER9), c (50CrMo4).
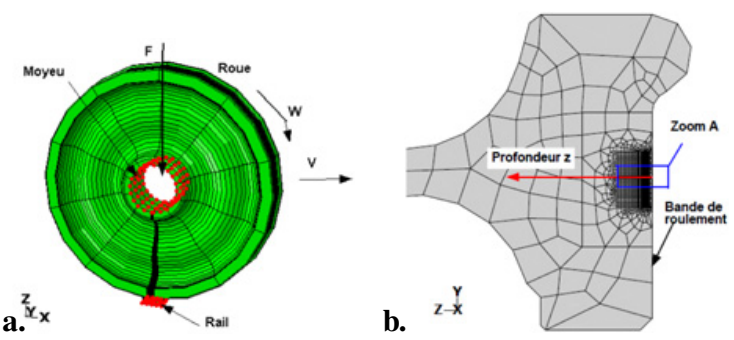

Figure 2. Maillage du modèle EF 3D : a-conditions aux limites, b-zone de contact pour les analyses en profondeur sous la bande de roulement.

Selon le chargement et la nuance d'aciers, on observe de différents types de réponses asymptotiques : élastique, adaptation élastique et accommodation plastique. Un récapitulatif des résultats est présenté dans le tableau cidessous : ELAS : réponse élastique, ADE : adaptation élastique, ACP : accommodation plastique.

L'acier 50CrMo4 présente des caractéristiques mécaniques meilleures, suivi de l'acier ER9, puis de l'acier ER7. a.

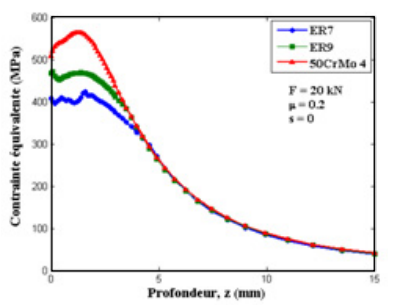

b.
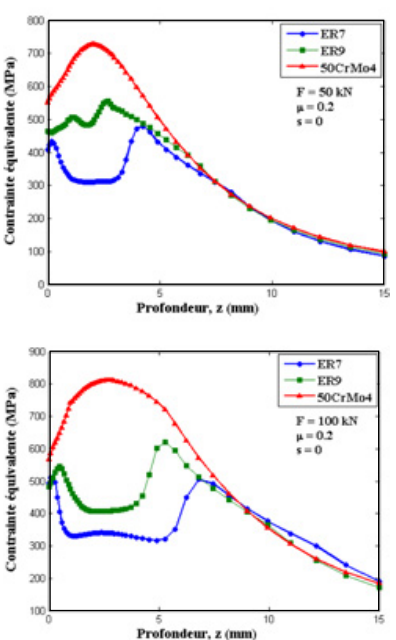

Figure 3. Evolution des contraintes de von Mises en profondeur, en roulement pur : a- Acier ER7, b- Acier ER9, c- Acier $50 \mathrm{CrMo} 4$.
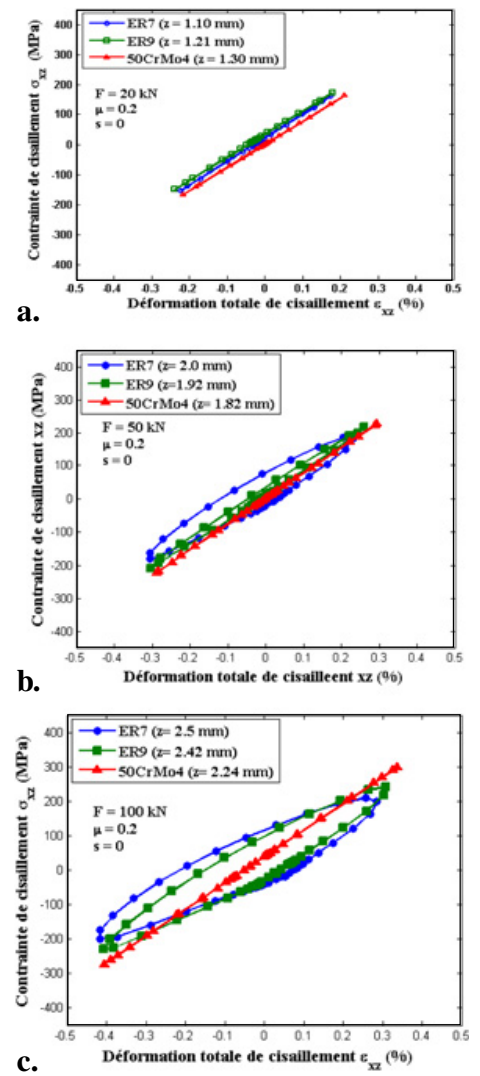

Figure 4. Boucles contrainte/déformation : a-20 kN, b- 50 kN, c$100 \mathrm{kN}$. 
Tableau 1. Récapitulatif des résultats: réponse asymptotique de la structure.

\begin{tabular}{|l|l|l|l|}
\hline $\mathrm{F}$ & ER7 & ER9 & $50 \mathrm{CrMo} 4$ \\
\hline $20 \mathrm{kN}$ & ADE & ADE & ELAS \\
\hline $50 \mathrm{kN}$ & ACP & ACP & ADE \\
\hline $100 \mathrm{kN}$ & ACP & ACP & ADE \\
\hline
\end{tabular}

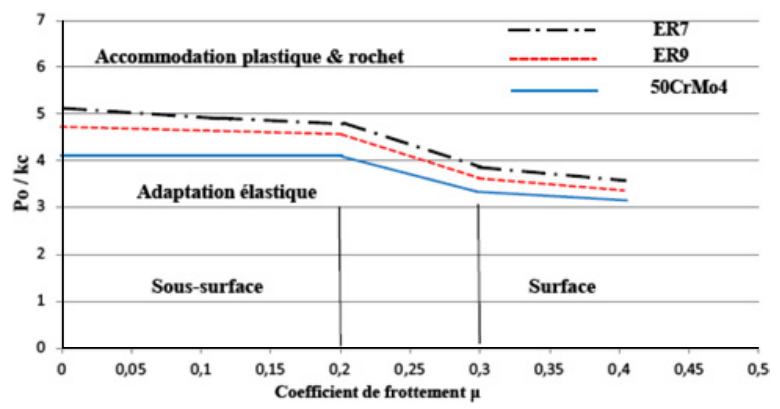

Figure 5. Limite d'adaptation.

\section{Influence de la nuance d'aciers sur la limite d'adaptation}

La limite d'adaptation de la roue est obtenue en conditions de glissement total de $10 \%$. La mise en œuvre des limites d'adaptation a nécessité plusieurs calculs à différent coefficient de frottement $(0$ à 0,5$)$ et à différentes charges $(10$ à $200 \mathrm{kN})$. La figure 5 montre une comparaison des limites d'adaptations des nuances d'aciers. Pour un coefficient de frottement $\mu=0$, les valeurs du facteur de charge $\mathrm{Po} / \mathrm{kc}=5,2$ pour le $\mathrm{ER} 7, \mathrm{Po} / \mathrm{kc}=4,78$ pour le ER9 et $\mathrm{Po} / \mathrm{kc}=4,2$ pour le $50 \mathrm{CrMo} 4$. Le facteur de charge de l'acier ER7 est plus élevé, mais la charge limite d'adaptation F est de $50 \mathrm{kN}$. En revanche le $50 \mathrm{CrMo} 4$ qui présente le plus petit facteur de charge, mais s'adapte élastiquement jusqu'à une charge de $200 \mathrm{kN}$. Les résultats montrent donc que le facteur de charge tel que défini dans les shakedown maps est insuffisant pour choisir acier. Il est donc nécessaire d'effectuer des analyses en fatigue.

\section{Influence de la nuance d'acier en fatigue de contact de roulement}

Les critères de Dang Van [4] et de Manson-Coffin [5] sont utilisés pour l'analyse en fatigue en vue de la comparaison des nuances d'aciers. Compte tenu des données matériaux disponibles pour le modèle de Dang Van, les aciers ER7 et 50crMo4 sont étudiés. La figure 6 représente les trajets de chargement en fatigue et les limites de Dang Van pour une charge de $20 \mathrm{kN}$. On remarque que la nuance d'aciers n'influence pas la résistance en fatigue, puisque les trajets de chargement ont le même niveau de criticité, mais sur l'adaptation de la roue comme montré auparavant.

Pour des charges entrainant l'accommodation plastique de la structure, le critère de Manson-Coffin a été utilisé pour estimer la durée de vie moyenne. La figure 7 présente l'amplitude de déformation plastique équivalente pour les trois nuances d'acier pour un coefficient de frottement $\mu=0,2$. La valeur maximale est localisée en sous-surface et varie en fonction de l'acier.

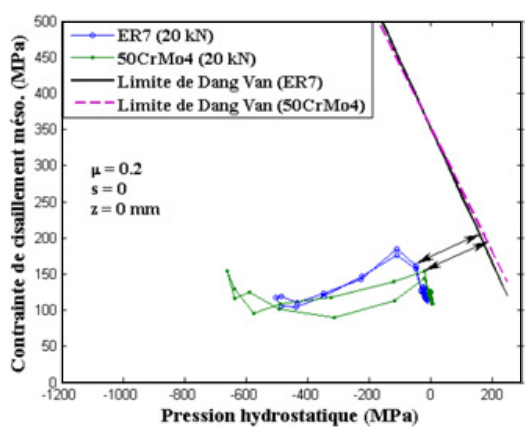

Figure 6. Trajet de chargement en fatigue au point critique en roulement pur et $\mathrm{F}=20 \mathrm{kN}$.

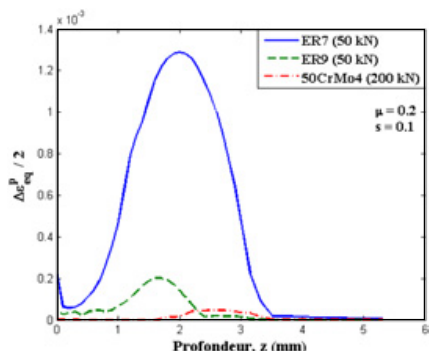

Figure 7. Evolution de l'amplitude de déformations plastiques en profondeur.

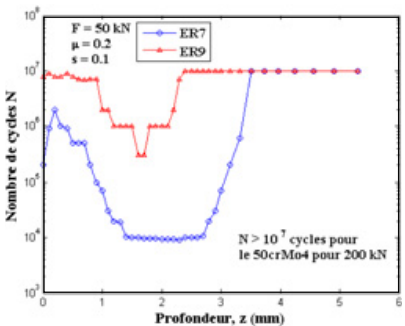

Figure 8. Durée de vie moyenne estimée en profondeur sous la bande de roulement.

La durée de vie moyenne correspondante aux trois aciers est présentée sur la figure 8. A noter qu'il est délicat d'estimer la durée de vie en surface dans la zone où l'usure sera importante. Néanmoins, les analyses que nous avons effectuées permettent d'avoir une idée sur l'usure en surface, dû au glissement dans le contact roue/rail.

\section{CONCLUSION}

L'influence de la nuance d'aciers en FCR a été étudiée dans ce travail. La méthode de dimensionnement adoptée permet de prendre en compte les sollicitations locales via la géométrie réelle du contact roue/rail, le comportement inélastique du matériau de la roue (acier) et intègre un critère de fatigue. L'étude a été centrée sur l'évolution de la réponse asymptotique de la structure, de sa la limite d'adaptation, de la résistance en fatigue et l'estimation de la durée de vie moyenne. Les résultats ont montré que le 50CrMo4 présente des propriétés mécaniques meilleures par rapport aux aciers de traitement thermiques ER7 et ER9. 


\section{Références}

[1] K.L. Johnson, J.A. Jefferis, Symp. Fatigue Rolling Contact 54, Institution of Mechanical Engineers, London, 1963.

[2] A. Langueh, J.-F. Brunel, E. Charkaluk, P. Dufrenoy, J.-B. Tritsch and F. Demilly: Effects of sliding on rolling contact fatigue of railway wheels, Wiley Publishing Ltd. Fatigue Fract Engng Mater Struct 36, 515-525, 2012.
[3] P.J. Armstrong, C.O. Frederick, Technical Report RD/B/N731, Berkeley Nuclear Laboratories, 1966.

[4] K. Dang Van, Macro-micro approach in high-cycle multiaxial fatigue, in D.L. McDowell and R. Ellis (eds), Adavances in multiaxial fatigue, ASTM STP 1991, American Society for testing and Materials, Philadelphia, 1993, 120-130.

[5] S.S. Manson, Behavior of materials under conditions of thermal stresses, TN, 2933, NACA, 1953. 